AperTO - Archivio Istituzionale Open Access dell'Università di Torino

\title{
A simple and reliable method to perform biomechanical evaluation of postoperative nerve adhesions.
}

\section{This is the author's manuscript}

Original Citation:

Availability:

This version is available http://hdl.handle.net/2318/1506160

since 2016-06-09T15:22:12Z

Published version:

DOI:10.1016/j.jneumeth.2014.06.008

Terms of use:

Open Access

Anyone can freely access the full text of works made available as "Open Access". Works made available under a Creative Commons license can be used according to the terms and conditions of said license. Use of all other works requires consent of the right holder (author or publisher) if not exempted from copyright protection by the applicable law. 


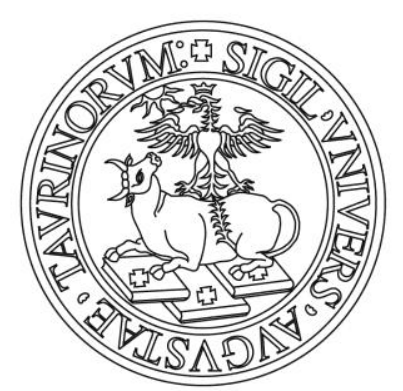

\section{UNIVERSITÀ DEGLI STUDI DI TORINO}

Thisis an authorversionof the contribution published on:

Questa è la versione dell'autore dell'opera:

J Neurosci Methods. 2014 Aug 15;233:73-7. doi:

10.1016/j.jneumeth.2014.06.008. Epub 2014 Jun 14.

The definitive version is available at:

La versione definitiva è disponibile alla URL:

http://www.sciencedirect.com/science/article/pii/S01650270140021

43 


\section{A SIMPLE AND RELIABLE METHOD TO PERFORM BIOMECHANICAL EVALUATION OF POSTOPERATIVE NERVE ADHESIONS}

Alessandro Crosio ${ }^{a}$, Luigi Valdattab, Mario Cherubino ${ }^{b}$, Matteo Izzo ${ }^{b}$, Igor Pellegattab, Davide Pascalc, Stefano Geunac, Pierluigi Tos ${ }^{a}$

a Reconstructive Microsurgery Unit, Traumatology Department, CTO Hospital, Via Zuretti 29, 10100 Turin, Italy

b Plastic Surgery Unit, Department of Biothecnologies and Sciences of Life, University of Insubria/Varese, Via JH Dunant 3, 21100 Varese, Italy

cHuman Anatomy Laboratory, Clinical and Biological Sciences Department, University of Turin, AOU San Luigi Gonzaga, Regione Gonzole 10, 10043 Orbassano, Italy

CORRESPONDING AUTHOR: Stefano Geuna, Anatomy Unit, Clinical and Biological Sciences Department, University of Turin, AOU San Luigi Gonzaga, Regione Gonzole 10 10043 Orbassano (TO), Italy

stefano.geuna@unito.it

+393397878055 


\section{ABSTRACT}

Background: Perineural fibrotic adhesions are among the major complications of peripheral nerve surgery. While different experimental models have been used for the pre-clinical testing of anti-adherential strategies, the methods used so far to induce scar tissue appear to be poorly standardized and reproducible.

New Method: Thirty adult mice were used. Two methods were tested: the first one is based on burning the perineural muscular bed with a diathermocoagulator, while the second is based on direct scratching of the nerve surface with a cotton swab. After 3 weeks, the fibrotic reaction was assessed by measuring the peak pull out force of the nerve from muscular bed by means of a new tool specifically devised for biomechanical assessment of scar tissue formation. Moreover, histological analysis with specific collagen stain was also carried out.

Results: both methods produced fibrotic reaction. Statistical analysis of biomechanical data showed a significant difference between burning and scratching group compared to the control sham operated group. No significant differences were detected between burning and scratching group. Histological analysis showed the presence of perineural scar tissue in both groups, though with a different distribution pattern.

Comparison with other methods: this protocol is easier to perform. The tool used for biomechanical evaluation is reliable and cheap.

Conclusions: both methods for perineural scar formation are effective and simple. They represent reproducible models for the study of the anti-adherential strategies. Yet, biomechanical testing with the device that we have developed proved to be a reliable and simple method for the quantitative assessment of the degree of perineural adhesion formation.

\section{KEYWORDS}

Nerve adhesion; perineural fibrosis; post surgical adhesion; compressive neuropathy. 


\section{INTRODUCTION}

Postsurgical perineural scar is one of the most frequent causes of compressive nerve syndromes (Jones et al 2012). To prevent this pathological condition, various treatment strategies have been proposed as barriers against perineural adhesions that can be applied after nerve surgery (Smit et al 2004, Ilbay et al 2004, Dam-Hieu et al 2005, Abe et al 2005, Yamamoto et al 2009). Whereas various techniques and protocols have been described to induce a perineural scar in experimental models for the preclinical testing of anti-adherence procedures, a recognized standardized protocol has not been defined yet (Smit et al 2004). This makes it difficult to compare the efficacy of the different anti-adherence strategies in order to optimize clinical treatment.

Two frequently used methods to induce scar formation can be found in the literature: the first one consists in a direct lesion (mechanical, thermal or chemical) applied to the surface of the nerve (Smit et al 2004, Dam-Hieu et al 2005, Zuijdendorp et al 2008); the second one consists in inducing a lesion of surrounding muscular bed which indirectly causes a damage to the nerve surface (Ikeda et al 2003, Abe et al 2005, Yamamoto et al 2009).

Moreover, two different methods have been commonly used for the evaluation of perineural scar: morphological analysis, either macro and/or microscopic (Park et al 2011, Ilbay et al 2004, Dam-Hieu et al 2005, Abe et al 2005, Yamamoto et al 2009) and functional biomechanical analysis (Ikeda et al 2003, Smit 2004, Abe et al 2005, Zuijdendorp et al 2008, Yamamoto et al 2009). Finally, also regarding animal models, there is no homogeneity in the literature since different species have been used so far (Ikeda et al 2003, Abe et al 2005, Zuijdendorp et al 2008, Yamamoto et al 2009).

In order to identify a shared model for postsurgical perineural scar investigation, we aimed to develop a reproducible and standardized protocol in the mouse sciatic nerve model. To this end, we have developed a simple tool for 
biomechanical analysis that we associated to histological assessment in order to obtain both quantitative and qualitative description of scar distribution pattern. 


\section{MATERIALS AND METHOD}

All procedures performed were in accordance with the Local Ethical Committee and the European Communities Council Directive of 24 November 1986 (86/609/EEC).

Thirty male mice (5 weeks old, average weight 28g, Charles River Laboratories, Lecco, Italy) were used in this study. After intra - peritoneal anesthesia with Ketamine $100 \mathrm{mg} / \mathrm{Kg}+$ Xylazine $15 \mathrm{mg} / \mathrm{Kg}$, under microscopic magnification, both sciatic nerves were exposed by gluteal splitting incision to view clearly the sciatic nerve from the gluteal vein to trifurcation (Fig. 1a). Then, each nerve was randomly assigned to one of the three experimental groups: burning group (1), scratching group (2), control group (3).

Burning group: after retraction of the nerve the muscle surface was burnt with diatermocoagulator for about $0.8 \mathrm{~cm}$ along the nerve bed (Fig 1b), as previously described (Ikeda et al 2003, Abe et al 2005, Yamamoto et al 2009).

Scratching group: with a cotton brush, 20 bites were made on the external surface of the nerve for about $0.8 \mathrm{~cm}$ (Smit et al 2004, Dam-Hieu et al 2005, Zuijdendorp et al 2008). The technique is illustrated in Fig 1c.

Control group: the nerve was exposed and the skin was closed immediately after.

Animals were housed with standard light conditions and unlimited access to food and water. After 3 weeks all animals were sacrificed by cervical dislocation. In each group, biomechanical evaluation was performed. Three nerves for each group were not tested biomechanically in order to be processed for histological evaluation.

Biomechanical evaluation was performed to measure the peak pull out force of the nerve from the muscular bed. An original instrument adapted from previously ones was developed (Figure 2a) (Ikeda et al 2003, Smith et al 2004, Abe et al 2005, Zuijdendorp et al 2008, Yamamoto et al 2009). The sciatic nerve was exposed near to the origin and loaded with 9-0 Nylon suture at the beginning of scar tissue (Fig 2b). 
Then, the suture was connected to a plastic can by a simple knot. The proximal end of the sciatic nerve was cut to 9-0 suture. Afterwards, the distal end of the sciatic nerve was exposed and cut.

The traction on the nerve increased gradually by means of a constant water flow filling up the plastic can. The water flow was kept constant at $100 \mathrm{ml} / \mathrm{min}$ and it was stopped when the nerve was definitely detached from his muscular bed. The peak pull out force was measured as the total weight of plastic can filled by water.

For histological analysis, the posterior space of the tight with nerve and scar tissue inside the muscles was harvested en bloc. The proximal end was marked with 9-0 Nylon. After paraffin inclusion (Raimondo et al, 2009), transversal sections $(11 \mu \mathrm{m}$ thickness) were obtained and stained with Sirius Red following previously described protocol (Carriel et al 2011).

Statistical analysis of results was made using the ANOVA test. 
RESULTS

Results of the biomechanical analysis are reported as the mean weight necessary to tear the nerve away from the muscle (Fig 3). Both methods for inducing scar tissue formation increased adhesion between nerve and muscles. Statistical analysis showed that both methods were fibrogenic in comparison to controls with high numerical significance (control vs scratching $\mathrm{p}<0.0001$, control vs burning $\mathrm{p}<0.0001$ ). By contrast, no statistical difference was detected between scratching group and burning group $(p>0.05)$.

Histological analysis confirmed the presence of scar tissue in both lesion groups. The sciatic nerve was stained in red such as the scar tissue surrounding the epineurium. In the control group there was not scar tissue (Fig. 4a). In burning group (Fig. 4b), perineural scar was strictly connected to the muscle and penetrated the epimisium. Therefore, the nerve appears embedded in the surrounding tissues. In the scratched group (Fig. 4c), scar tissue affected, as expected, mainly the epineurium that appears thickened, with less involvement of the surrounding muscle bed. 


\section{DISCUSSION}

Although a number of experimental techniques have been described to produce perineural scar tissue (Smit et al 2004, Dam-Hieu et al 2005, Zuijdendorp et al 2008 Ikeda et al 2003, Abe et al 2005, Yamamoto et al 2009 Park et al 2011, Ilbay et al 2004), a standardized and reproducible method is still not widely recognized. Since the availability of a standardized and shared method to create and study perineural scar formation is very important to test which anti adherential products/devices are more effective in the clinical perspective, the aim of our work was to define a simple, cheap and reliable model to evaluate adhesion between the nerve and surrounding muscle in the mouse sciatic nerve model.

To this end, the two most used methods to induce perineural scar formation were compared in this study, namely scratching nerve surface (Smit et al 2004, DamHieu et al 2005, Zuijdendorp et al 2008) and burning the muscle bed (Ikeda et al 2003, Abe et al 2005, Yamamoto et al 2009). Yet, a reliable biomechanical method for evaluating scar tissue quantitatively was developed in order to obtain data that allow the comparative assessment of anti-adherential treatment strategies.

Although several authors used histological evaluation to define scar tissue formation degree (Park et al 2011, Ilbay et al 2004, Dam-Hieu et al 2005, Abe et al 2005, Yamamoto et al 2009), in a pilot study phase we ruled out the possibility to use histological evaluation to obtain quantitative measurements of scar formation. In fact, in our model histology is adequate to describe and illustrate the distribution and location of the scar tissue formation, but not to assess quantitatively its degree because of the high variability in the histological appearance that can be found in different slices of the same nerve. 
On the other hand, we found out that biomechanical analysis provides reliable quantitative data by means of a simple dedicated tool that we have devised. The tool is inspired to the one described by Smit et al (2004), in comparison to which is simpler and cheaper.

However, it should be emphasized that, in order to obtain a realistic interpretation of perineural scar tissue, it is necessary to assess its entity from different points of view and using different and complimentary techniques, including clinical observation and histological analysis. In this context, biomechanical assessment only represents one of the methods for investigating scar tissue formation in peripheral nerves.

The importance of careful surgical handling should be emphasized. First, during the primary surgical procedure it is important to avoid bleeding for avoiding the unpredictable fibrogenic activity of blood. At time of tissue harvesting, it is important not to pull the nerve with forceps and to anchor the nerve for loading it just at the margin of scar tissue. Finally, it is necessary to be meticulous in avoiding the formation of nerve bundles which can interfere with traction testing.

As far as the comparison between the two different protocols for inducing scar formation is concerned, our results did not reveal any significant biomechanical difference between them, whereas both of them are able to induce significant adhesions compared to control group. Hence, both methods can be regarded as effective for investigating perineural scar tissue formation. However based on the additional qualitative histological assessment, we support the view that the best method according to both analysis is to burn the muscle. In fact, this method creates more diffuse adherence all around the nerve without directly damage to the structure of the nerve. Furthermore, burning scar tissue has hemostatic effect and thus prevents bleeding that has unpredictable fibrogenic effect that can increase variability among animals.

Finally, while rats (Zuijdendorp et al 2008, Yamamoto et al 2009) and rabbits 
(Ikeda et al 2003, Abe et al 2005) have been mainly used so far for nerve scar investigation, we were able to show that methods for scar induction are adaptable also to the mouse, namely the most widely used rodent experimental model, opening interesting research perspectives due to the large availability of genetically modified mice (Tos 2008).

Although our biomechanical results are similar to those obtained in previous studies that applied biomechanical evaluation to assess perineural scar formation in larger animal models (Ikeda et al 2003, Smit et al 2004, Yamamoto et al 2009), it should be pointed out that we registered higher standard deviation in all groups due to the smaller size of the nerve in mice and consequent lower tensile strength. However, in spite of the higher variance, our set of biomechanical data allowed to detect significant differences between groups and the experimental protocol will thus allow to assess the effectiveness of various anti-adherential treatments, such as chemical agents and adipose-derived stem cells.

\section{CONCLUSION}

We describe a simple and reliable tool for the evaluation of perineural scar tissue in the mouse sciatic nerve.

Biomechanical analysis gives us a quantitative evaluation of scar tissue. However, to have a correct interpretation of scar tissue formation is necessary to associate also histological analysis that may show different patterns of scar distribution.

This simple tool can be used to compare different anti-adherence strategies before their clinical translation and to validate their efficacy quantitatively. 


\section{REFERENCES}

Jones NF, Ahn HC, Eo S. Revision Surgery for Persistent and Recurrent Carpal Tunnel Syndrome and for Failed Carpal Tunnel Release. Plastic and Reconstructive Surgery 2012;129(3):683-692

Smit X, Van Neck JW, Afoke A, Hovius SER. Reduction Of Neural Adhesions By Biodegradable Autocrosslinked Hyaluronic Acid Gel After Injury Of Peripheral Nerves: An Experimental Study. J Neurosurg 2004;101:648-652

Dam-Hieu P, Lacroix C, Said GR, Devanz P, Liu S, Tadie M. Reduction of Postoperative Perineural Adhesions by Hyaloglide Gel: An Experimental Study in the Rat Sciatic Nerve. Neurosurgery 2005; 56(Supplement 2):425-433

Zuijdendorp HM, Smit X, Blok JH, Caruelle JP, Barritault D, Hovius SER, Van Neck JW. Significant reduction in neural adhesions after administration of the regenerating agent OTR4120, a synthetic glycosaminoglycan mimetic, after peripheral nerve injury in rats J Neurosurg 2008;109:906007-900703

Ikeda K, Yamauchi D, Osamura N, Hagiwara N, Tomita K. Hyaluronic acid prevents peripheral nerve adhesion. British Journal of Plastic Surgery 2003;56(4): 342-347

Abe Y, Doi K, Kawai S. An experimental model of peripheral nerve adhesion in rabbits. British Journal of Plastic Surgery 2005;58(4): 533-540

Yamamoto M, Endo N, Ito M, Okui N, Koh S, Kaneko H, Hirata H. Novel Polysaccharidederived hydrogel prevents perineural adhesions in a rat model of sciatic nerve adhesion. Journal of Orthopaedic Research 2009;28:284-288 
Park JS, Lee JH, Han CS, Chung DW, Kim GY. Effect of Hyaluronic AcidCarboxymethylcellulose Solution on Perineural Scar Formation after Sciatic Nerve Repair in Rats. Clinics in Orthopedic Surgery 2011;3(4): 315-324

Güzin Y, Im Ö, Gülaydan F. Effects of human amniotic fluid on peripheral nerve scarring and regeneration in rats. J Neurosurg 2003;98: 371-377

Carriel V, Garzón I, Alaminos M, Campos A. Evaluation of myelin sheath and collagen reorganization pattern in a model of peripheral nerve regeneration using an integrated histochemical approach. Histochem Cell Biol 2011;136(6):709-17

Raimondo S, Fornaro M, Di Scipio F, Ronchi G, Giacobini-Robecchi MG, Geuna S. Methods and protocols in peripheral nerve regeneration experimental research. Part IIMorphological techniques 2009;87:81-103.

Tos P, Ronchi G, Nicolino S, Audisio C, Raimondo S, Fornaro M, Battiston B, Graziani A, Perroteau I, Geuna S. Employment of the mouse median nerve model for the experimental assessment of peripheral nerve regeneration. J Neurosci Methods 2008;169:119-127 


\section{FIGURE CAPTIONS}

Fig 1: microscopical view of the gluteal splitting approach (a). Burn injury of muscle bed with diathermocoagulator (b). Scratch injury of sciatic nerve using cotton swab.

Fig 2: extraction tool to perform biomechanical evaluation. In panel a, the microscopical view of the proximal sciatic nerve end connected to cable that sustain the plastic can is present6ed. A schematic view of the entire extraction tool is presented in panel b. After nerve transection at the knee and proximal to injury site, the traction on the nerve is increasing using a constant water flow from bottle to container at $100 \mathrm{~mL} / \mathrm{min}$. The weight of container at detachment moment is recorded as peak pull out force that correspond to scar duress.

Fig 3: results of biomechanical assessment. Burning group presents the highest peak pull out force although no statistical difference was detected between the two lesion group. Otherwise both methods can induce scar tissue compared to control group.

Fig 4: Histological view of transvers section of en bloc withdrawal (Sirius Red Stain, 10x). In panel a, normal aspect of sciatic nerve (S) and his surrounding muscles without pathological scar tissue is shown. After burning of muscle bed (b), scar tissue in identifiable $(*)$, collagen fibers spiculae $(\rightarrow)$ strictly connect nerve to surrounding tissue. After scratching injury (c) scar tissue in also appreciable $\left({ }^{*}\right)$, but in contrast to the other method there is no invasion of muscle bed. 